XXVIII.

\title{
Transitorische Hemianopsie und concentrische Gesichtsfeldeinschränkung bei einem Falle von cerebraler Kinderlähmung*).
}

Von

\author{
Dr. Wilhelm Koenig, \\ Oberarzt an der Irrenanstalt der Stadt Berlin zu Dalldorf.
}

\begin{abstract}
$D_{\text {i }}$ ie folgende Mittheilung betrifft ein jetzt 12 jähriges epileptisches Mädchen; es ist das ein Fall, welcher bereits vor einigen Jahren im hiesigen psychiatrischen Verein Gegenstand einer Krankenvorstellung von meiner Seite gewesen und später unter der Ueberschrift: „Ueber eine seltene Form der cerebralen Kinderlähmung" veröffentlicht worden ist**). Die Seltenheit der Form bestand, um es kurz zu recapituliren, darin, dass sich bei dem Kinde eine isolirte Parese des rechten Mundfacialis zeigte, welche als Rest einer im Uebrigen zurückgegangenen rechtsseitigen Hemiplegie anzusehen war und die Eigenthümlichkeit darbot, bei mimischen Bewegungen, namentlich beim Lächeln, mit besonderer Intensität zu Tage zu treten. Patientin litt ferner an epileptischen Anfällen allgemeiner Natur, welche aber, soweit ich dieselben selbst beobachten konnte, regelmässig eine passagere, einige Minuten anhaltende Parese der rechten Extremitäten zu hinterlassen pflegten.

In der Zwischenzeit hat Patientin einen schweren Gelenkrheumatismus durchgemacht und im April v. J. in einem Anfall eine Infraction der rechten Clavicula sich zugezogen. Die Anfälle treten in unbestimmten Tntervallen noch immer auf. Bezüglich der Lähmungserscheinungen hat sich nichts geändert.

Am 1. Januar v. J., als Patientin sich bei ihrer Mutter auf Urlaub befand, traten die Anfälle plöŁzlich in erheblicher Anzahl und mit grosser Inten-
\end{abstract}

*) Nach einem in der Berliner Gesellschaft für Psychiatrie und Nerrenkrankheiten am 11. März 1895 gehaltenen Vortrage.

**) Deutsche med. Wochenschrift. 1893. No. 42.

Archiv f. Psychiatrie. XXVII. 3. Hefi. 
sität auf: bis zu 25 innerhalb 24 Stunden, und hielten in dieser Weise bis zum 7. Januar an. Am 5. Januar, nachdem Patientin während der Nacht 4 Anfälle gehabt hatte, fiel es der Mutter Morgens früh, als das Kind sich ankleiden wollte, auf, dass es schlecht sah. Wollte Patientin einen Gegenstand erkennen, so musste sie den Kopf nach rechts drehen und etwas seitwärts beugen. In dieser Intensität soll die Sehstörung 8 Tage angehalten haben. Leider wurde während dieser Zeit keine ärztliche Hülfe in Anspruch genommen. Es ist das deswegen besonders zu bedauern, weil es von Interesse gewesen wäre, zu erfahren, ob während der Zeit Symptome von Seelenblindheit bezw. optischer Aphasie vorhanden gewesen sind. Erst am 10. Tage der Erkrankung, am 15. Januar, als die Sehstörung sich bereits gebessert hatte, insofern wenigstens Patientin die vorhin geschilderte Kopfbewegung nicht mehr zu machen brauchte, um einen Gegenstand zu erkennen, ging die Mutter mit dem Kinde nach der Augenklinik des Herrn Dr. Wertheim. Herr College W. hatte die Liebenswürdigkeit, mir nachträglich über seine Untersuchong Mittheilung zu machen.

Bei grober Untersuchnng fand er auf dem rechten Auge einen Ausfall der ganzen rechten Gesichtsfeldhälfte, die Trennungslinie ging genau durch den Fixationspunkt; links: Ausfall eines Theiles der inneren Gesichtsfeldhälfte. $S=1 / 2$. Ophthalmoskopisch normal. Gleich nach dieser Untersuchung bekam Patientin einen rudimentären Anfall, so dass von einer perimetrischen Aufnahme Abstand genommen werden musste. Am 17. Januar wurde Patientin uns wieder zugeführt; am 18. Januar sah ich sie zuerst und stellte zunächst bei grober Untersuchung das Bestehen einer rechtsseitigen homonymen Hemianopsie fest. Es fiel mir dabei jedoch auf, dass die Trennungslinie nicht mehr durch den Fixationspunkt ging, sondern etwas davor fiel.

Patientin orientirte sich gut im Raume, hatte keine subjectiven Beschwerden mehr. Optische Aphasie war nicht vorhanden. S. $=1 / 2$. Ophthalmoskopischer Befund normal.

Am 19. Januar wurde die erste einer grösseren Anzahl perimetrischer Anfnahmen gemacht, durch welche eine interessante fortschreitende Umgestaltung des Gesichtsfeldes festgestellt wurde.

Die Untersuchungen wurden am Förster'schen Perimeter mit einem Untersuchungsobject von $5^{2} \mathrm{Mm}$. ausgeführt. Die Aufmerksamkeit und Ausdauer der kleinen Patientin bei den Untersuchungen war eine musterhafte.

Auf Gesichtsfeld I. (yom 19. Januar) sieht man zunächst eine rechtsseitige homonyme Hemianopsie mit concentrischer Einschränkung des restirenden Gesichtsfeldes, und zwar ist die Einschränkung rechts stärker als links; weiter bemerkt man, dass die Trennungslinie, wie ich bereits bei grober Untersuchung festgestellt hatte, nicht durch den Mittelpunkt geht, denn es findet sich auf beiden Arigen ein von der Gegend des Fixationspunktes aus in horizontaler Richtung in die hemianopischen Gesichtsfeldhälften sich erstreckendes schmales Stück orhaltenen Gesichtsfeldes, rechts geht dies bis zum 8. ${ }^{0}$, links bis zum $20 .{ }^{0}$.

Bekanntlich ist es bei Hemianopsien sehr häufig der Fall, dass die Trennungslinie nicht durch den Fixationspunkt geht, sondern dass das erhaltene 
Gesichtsfeld I.

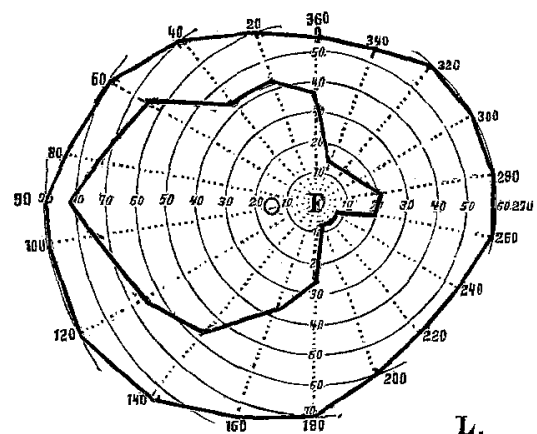

L.

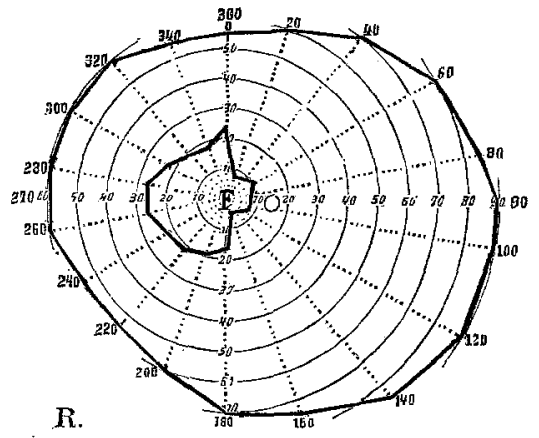

Gesichtsfeld II.
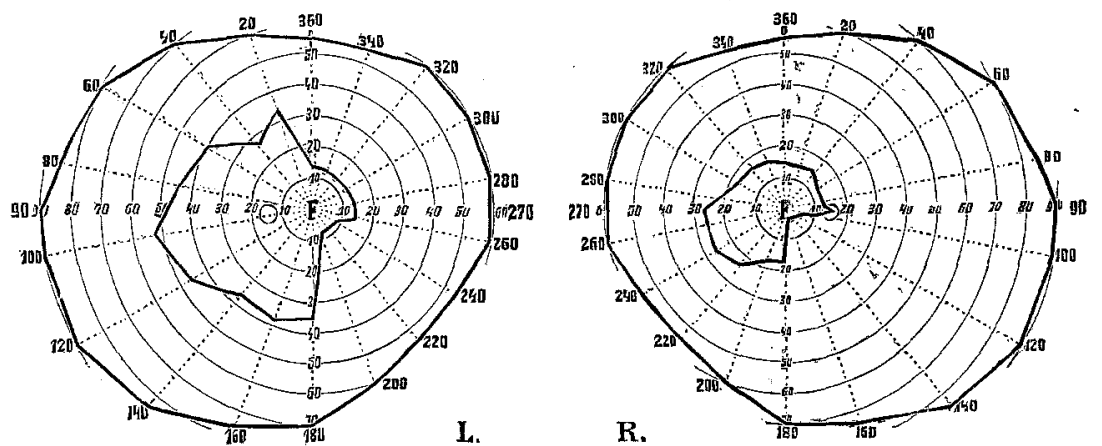

Gesichtsfeld III.
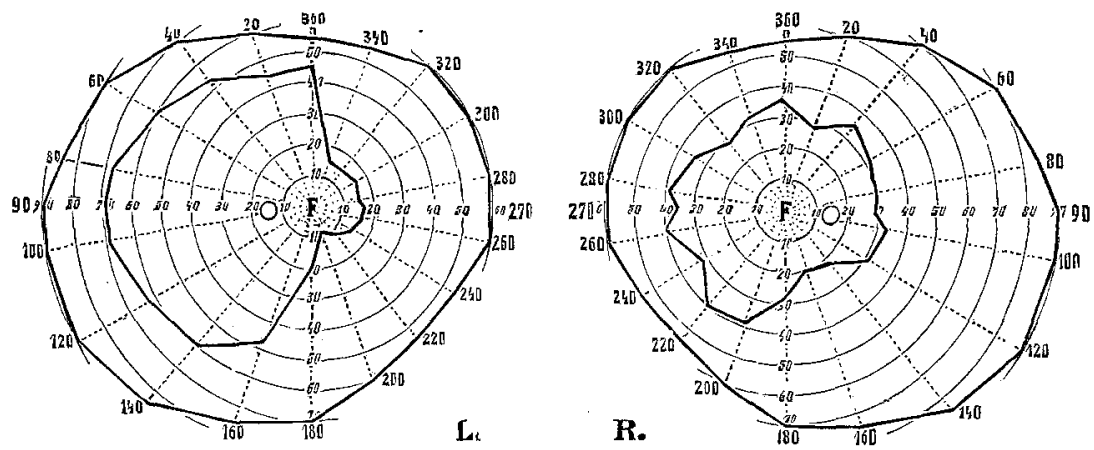

Gesichtsfeld etwas in die hemianopische Hälfte hinübergreift (nUeberschüssiges Gesichtsfeld“). In diesem Falle handelt es sich aber nicht um "überschüssiges Gesichtsfeld"; dagegen spricht vor Allem die Ausdehnung des in die hemian- 
opische Hälfte hineinragenden Stïckes erhaltenen Gesichtsfeldes, namentlich auf dem linken Auge, ferner die Thatsache, dass einige Tage vor dieser Aufnahme die Trennungslinie nach der Untersuchung des Herrn Collegen Wertheim durch den Fixationspunkt ging; das Gesichtsfeld hatte sich also seitdem verändert, und zwar haben wir in dieser Veränderung, wie dies bis zur Evidenz aus den späteren Aufnahmen hervorgeht, die beginnende Rückbildung der Hemianopsie zu erblicken.

Auf Gesichtsfeld II. (20. Januar 1895) sieht man den weiteren Rü̈ckgang der Hemianopsie, rechts mehr als links, rechts kann man fast schon von einer Quadranthemianopsie sprechen; im auffälligen Gegensatz hierzu hat, worauf ich besonders aufmerksam machen möchte, die concentrische Einengung auf beiden Augen, speciell links, erheblich zugenommen.

Auf Gesichtsfeld III. (21. Januar 1895) ist der hemianopische Charalkter nur links noch auffällig; rechts hat das Gesichtsfeld schon mehr Aohnlichkeit mit einer gewöhnlichen concentrischen Einschränkung; sieht man aber näher hin, so ist die sich rückbildende Hemianopsie doch noch zu erkennen; die temporale Hälfte des erhaltenen Gesichtsfeldes ist nämlich kileiner als die nasale, was bekanntlich bei der functionellen concentrischen Gesichtsfeldeinschränkung' nicht vorkommt; auch der einspringende Winkel im äusseren unteren Quadranten verräth noch die abklingende Hemianopsie. Die allgemeine concentrische Einschränliung ist erheblich geringer wie anf Gesichtsfeld II., aber auch hier links stärker ausgesprochen als rechts.

Gesichtsfeld IV. (22. Januar 1895) ist principiell von III. nicht verschieden, nur sieht man die Hemianopsie aufeiner weitereren Stufe der Reevolution. Das Gesichtsfeld für grün *) zeigt auf dem rechten Auge den hemianopischen

Gesichtsfeld IV.
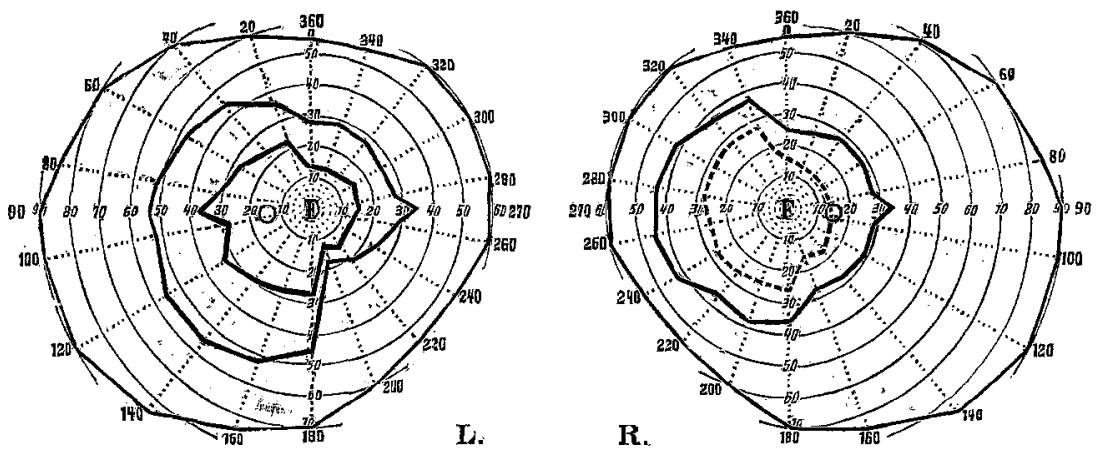

Charakter noch ganz deutlich. Die concentrische Einschränkung hat links wieder zugenommen. Da mir bis dahin die Bedeutung dieser Gesichtsfeldmetamorphose nicht sicher war, andererseits aber der Fall ein interessanter zu sein schien, hielt ich es für zweckmässig, Patientin noch von dritter, bis dahin

*) Grün $=\ldots$ 
unbetheiligter Seite, untersuchen zu lassen; ich schickte sie deshalb am 23. Januar nach der Augenpoliklinik des Herrn Prof. Schöler, wo Herr College Somya die Güte hatte, eine genaue Untersuchung vorzunehmen und mir folgendes Gesichtsfeld, welches ich als No. V. bezeichnet habe, zuzuschicken,

\section{Gesichtsfeld V.}
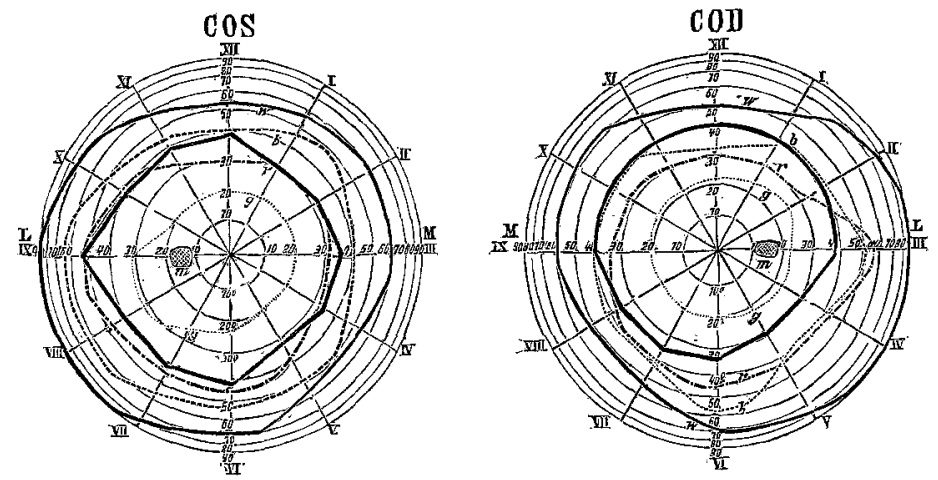

mit der Diagnose: "hysterische concentrische Gesichtsfeldeinschränkung""; wenn man dieses Gesichtsfeld betrachtet, so wird man wohl mit dieser Auffassung sich einverstanden erklären. Vergleicht man es aber wiederum mit den demonstrirten Gesichtsfeldern einerseits und andererseits mit den noch zu demonstrirenden, so sieht man, dass es auch vollständig in diese Rückbildungsreihe hineinpasst.

Die Ausdehnung der concentrischen Einschränkung zunächst ist fast ganz dieselbe wie auf Gesichtsfeld IV. Nur ist der hemianopische Charakter noch mehr verloren gegangen und in der That kaum noch zu erkennen. Rechts ist die temporale Hälfte des Gesichtsfeldes noch immer eine Spur kleiner als die nasale; die Differenz ist allerdings eine so minimale, dass ihr nur im Zusammenhang mit den anderen Gesichtsfeldern Bedeutung zugesprochen werden darf. Die Gesichtsfelder für blau, roth und grün, deren Einzeichnung hier weggelassen ist, waren beiderseits entsprechend dem G. F. für weiss eingeschränkt.

Das zwei Tage darauf am 25. Januar von mir aufgenommene Gesichtsfeld VI. zeigt ungefähr denselben Charalter, nur hat sich das Gesichtsfeld im Allgemeinen etwas erweitert. Die temporale Hälfte rechts zeigt ungefähr dieselbe Ausdehnung, wie die nasale.

In dem 8 Tage später, am 13. Februar aufgezeichneten Gesichtsfeld VII. haben wir zum ersten Male eine ganz typische concentrische Gesichtsfeldeinschränkung für weiss wie für Farben*); links hat sich das Gesichtsfeld noch mehr aufgehellt und rechts ist jetzt die temporale Hälfte deutlich grösser als die nasale.

Auf den nächsten Aufnahmen (Fig. VIII., IX. und X.) rom 23. Februar,

*) Die Gesichtsfelder für Farben sind, da sie nichts Besonderes zeigen, weggelassen worden. 
4. März und 10. März sehen wir das Gesichtsfeld allmälig bis zur Norm sich erweitern; auch für Farben war dies der Fall. Die Sehschärfe war am 4. März ca. $\frac{6}{9}$ am 10. März (Dr. Abels dor ff) $\frac{6}{6}$.

\section{Gesichtsfeld VI.}
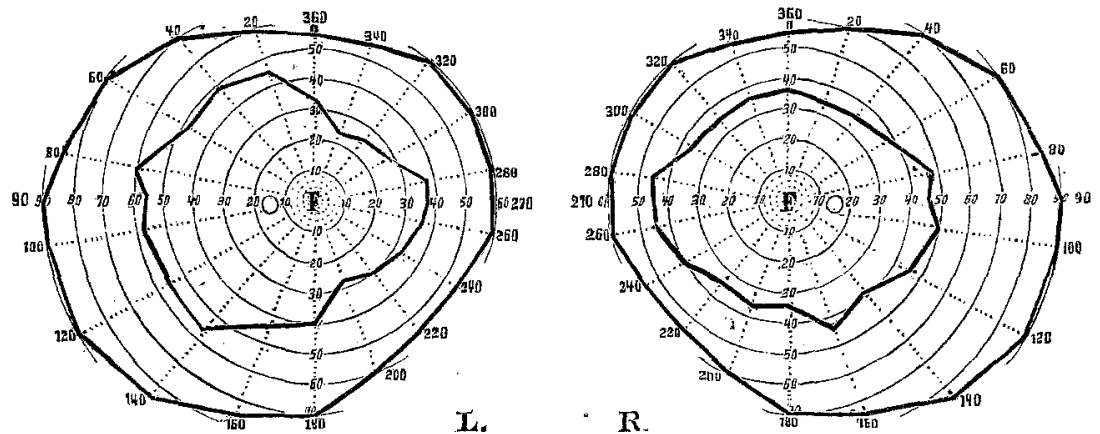

Gesichtsfeld VII.
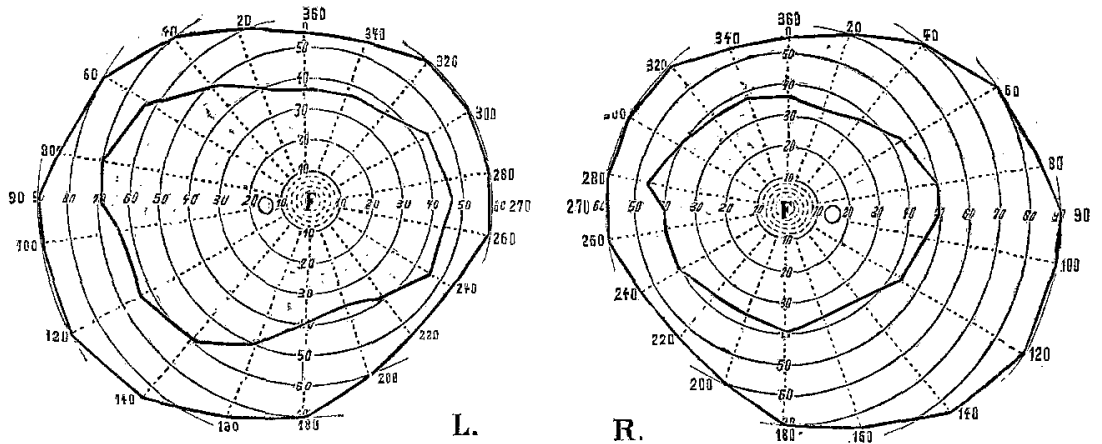

Gesichtsfeld VIII.
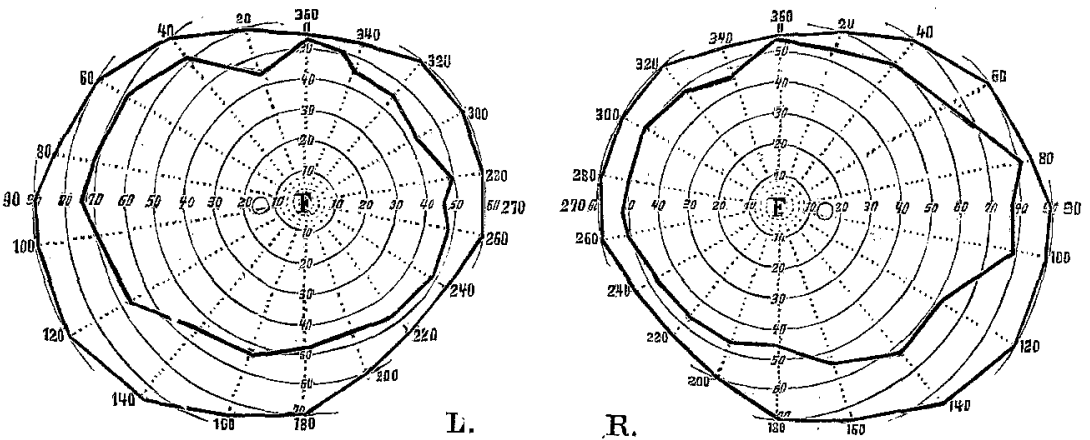
Gesichtsfeld IX.
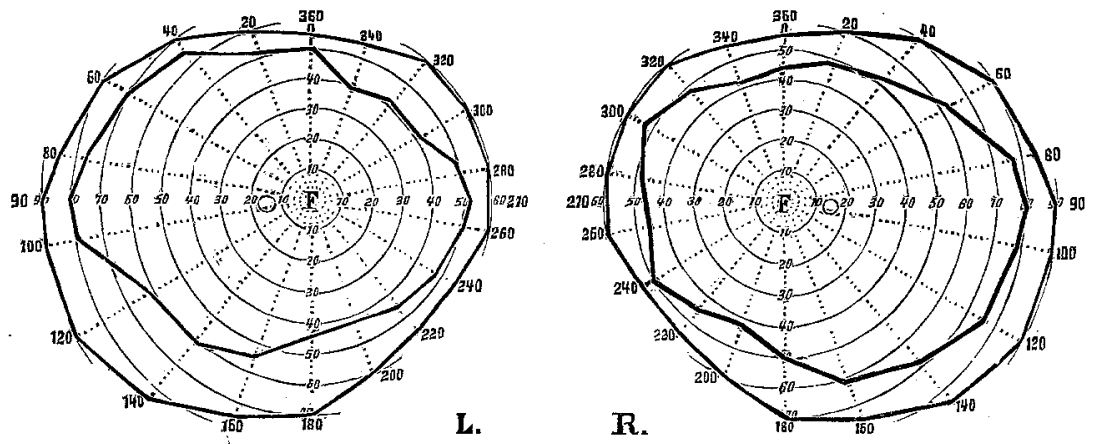

\section{Gesichtsfeld X.}

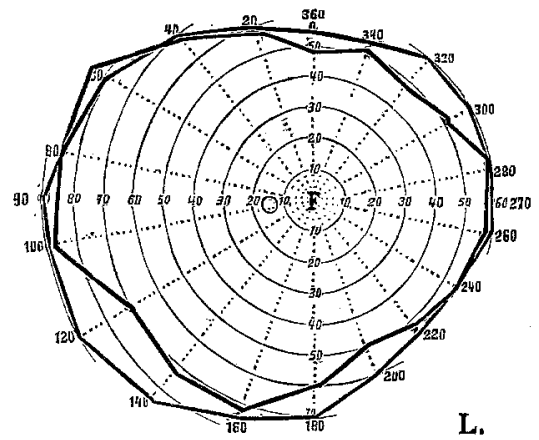

L.

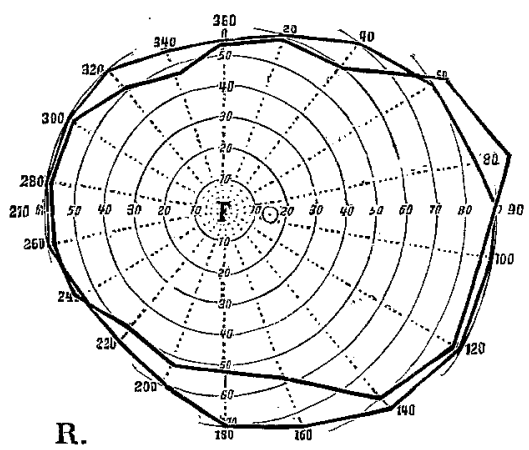

R.

Ich habe noch hinzuzufügen, dass Wilbrand'scher Ermüdungstypus sich während der ganzen Zeit der Beobachtung niemals nachweisen liess, dass, abgesehen ron einigen schmerzhaften Druckpunkten auf dem Sternum, keine weiteren hysterischen Stigmata vorhanden sind, dass Patientin seit ihrer Wiederaufnahme von Anfällen verschont geblieben ist und sich vollkommen wohl fühlt. -

Fassen wir das eben Gesagte kurz zusammen, so finden wir bei einem 12 jährigen an Epilepsie und unvollständiger rechtsseitiger Kinderhemiplegie leidenden Mädchen das acute Auftreten einer Sehstörung nach gehäuften Anfällen; diese Sehstörung findet ihren Ausdruck darin, dass Patientin gezwungen ist, Gegenstände, die sie erkennen will, in die äussere Hälfte ihres linken Gesichtsfeldes zu bringen. Die 14 Tage später vorgenommene perimetrische Untersuchung ergiebt zunächst eine im Beginn der Rückbildung sich befindende rechtsseitige Hemianopsie mit concentrischer Einschränkung des erhaltenen Gesichtsfeldes bei einer Sehschärfe ron $\frac{1}{2}$. Im Laufe der nächsten Wochen 
bildet sich das Gesichtsfeld allmälig zur Norm zurück, und zwar geht dieser Vorgang so vor sich, dass sich zunächst die Hemianopsie zurückbildet innerhalb des concentrisch eingeschränkten Gesichtsfeldes, dessen Grenzen während der Reevolution der Hemianopsie erheblichen Schwankungen unterliegen und erst nach derselben sich allmälig bis zur Norm erweitern. Auch die Sehschärfe wird nach und nach eine normale.

Wenn wir uns nun die Bedeutung der eben geschilderten Vorgänge klar machen wollen, so thun wir, glaube ich, gut, die Hemianopsie und die concentrische Gesichtsfeldeinschränkung gesondert zu betrachten.

Die Kenntniss von dem Vorkommen der Hemianopsie bei cerebraler Kinderlähmung ist noch relativ neueren Datums; die ersten Beobachtungen wurden von Freud gemacht und finden sich in der von ihm und $\mathrm{Rie}$ verfassten Monographie*); es werden da drei Fälle beschrieben, welche kleine Kinder betreffen, bei welchen natürlich eine perimetrische Untersuchung nicht angestellt werden konnte. Zwei weitere Fälle sind von Henschen**) beschrieben worden; ein sechster Fall wurde von Köppen im Jahre 1893 in der Gesellschaft der Charité-Aerzte***) vorgestellt; in diesem Falle wie in den beiden von Henschen fehlt auch die perimetrische Aufnahme nicht; damit ist, soweit ich die Literatur übersehe, die Casuistil erschöpft $\dagger$ ). In den eben angeführten Fällen handelte es sich um dauernde Ausfallserscheinungen $+\uparrow$ ). In der transitorischen Form ist die Hemianopsie bis jetzt bei cerebraler Kinderlähmung noch nicht beobachtet worden.

An und für sich begegnen wir ja transitorischen Hemianopsien nicht so selten. Wir finden sie zuweilen nach apoplectischen Anfällen, nach paralytischen Insulten, nach Krampfanfällen anderer Art, z. B. in Folge von Uränie --, ein sehr interessanter Fall dieser Art wurde von Köppen $\dagger \dagger \dagger$ ) beschrieben -, und als Theilerscheinung der Migraine ophthalmique; endlich findet sich eine isolirte Beobachtung von $\mathrm{Hoche}$ bei einer functionellen Psychose + ). Von den durch materielle, speciell gummöse Erkiankungen an der Hirnbasis bedingten vorübergehenden und oscillirenden Hemianopsien sehe ich hier ab.

*) Freud und Rie, Studien über die halbseitige Cerebrallähmung der Kinder. Wien 1891.

**) Klinische und anatom. Beiträge zor Pathologie des Gehims. Bd. T. Upsala 1890.

***) Verhandlungen der Gesellschaft der Charité-Aerzte. Berliner klin. Wochenschr. 1893. S. 900.

†) Anmerkung bei der Correctur: Sachs erwähnt ganz kurz, dass єr Hemianopsie $8 \mathrm{Mal}$ nachweisen konnte. (Sachs: „Die Hirnlähmungen der Kinder". Klin. Vorträge. Mai 1892.)

†) Ueber den dritten Fall von Freud fehlt noch die Angabe des weiteren Verlaufs.

†††) Charité-Annalen XVIII. Jahrgang.

*) Dieses Archir Bd. XXIII. 
Nach gewöhnlichen epileptischen Anfällen wird Hemianopsie nicht beobachtet; wird das Gesichtsfeld afficirt, so geschieht dies in der Form der concentrischen Einschränkung, welche sich allmälig wieder ausgleicht, wie dies u. A. in dem bekannten Falle von Pick*) in sehr exacter Weise beobachtet und geschildert worden ist.

Die Thatsache, dass in unserem Falle Hemianopsie nach gehäuften Anfällen auftrat, steht nur in scheinbarem Widerspruche mit der eben aufgestellten Behauptung. Ich hob zu Anfang hervor, dass die von mir beobachteten Anfälle sich insofern von gewöhnlichen epileptischen unterschieden, als sie eine passagere Lähmung der rechten Extremitäten zu hinterlassen pflegten, also gewissermassen als eine atypische Form der Rindenepilepsie aufgefasst werden konnten. Die Verhältnisse liegen nun so, dass während früher die linke motorische Zone die durch die Anfälle afficirte Gegend zu sein pflegte, es dies Mal der linke Occipitallappen gewesen ist, welcher in Mitleidenschaft gezogen wurde.

Beide Male dürften wohl die Ausfallserscheinungen functioneller Natur gewesen und auf Circulationsstörungen, event. auf andere feinere dynamische Veränderungen zurückzuführen sein.

Eine andere Erklärung erfordert die concentrische Gesichtsfeldeinschränkung; diese tritt, wie bekannt, häufig als Complication zu den auf anatomischen Veränderungen beruhenden Hemianopsien hinzu, wir sehen sie aber nicht als Herdsymptom an, sondern als eine functionelle Begleiterscheinung. Functionell hier in dem Sinne, in welchem wir diesen Ausdruck bei der Hysterie verwenden $* * *$ ).

Das gegenseitige Verhalten der Hemianopsie und der concentrischen Gesichtsfeldeinschränkung kann nun ein doppeites sein. Entweder verschwindet die letztere nach kürzerer oder längerer Zeit und der hemianopische Defect bleibt zurück, oder beide Symptome erweisen sich als stabil.

Was die Verhältnisse bei transitorischer Hemianopsie anbelangt, so liegen meines Wissens bis jetzt keine darauf gerichteten Untersuchungen vor. Es liegt das wohl zum Theil daran, dass Fälle, in welchen eine Verfolgung der sich innerhalb des Gesichtsfeldes abspielenden Vorgänge am Perimeter möglich ist, selten sind. Um so wünschenswerther muss es erscheinen, dass in Zukunft bei geeigneten Fällen auch auf diesen Punkt geachtet wird.

Was unseren Fall anbelangt, so liegt, wie mir scheint, kein Grund yor, die concentrische Einschränkung, welche sich in jeder Beziehung genau wie eine hysterische verhält, anders aufzufassen, als wir dies in Fällen von stabiler Hemianopsie zu thun gewohnt sind. Dass es sich um auf verschiedener Basis beruhende Vorgänge handelt, zeigt das Verhalten bei der Reevolution des Gesichtsfeldes ganz besonders deutlich; während nämlich die Rückbildung der Hemianopsie Schritt für Schritt vor sich ging, war das Verhalten der

*) Dieses Archir 1891. S. 756.

**) Natürlich schliesse ich auch hier die durch organische Erkrankungen des Opticus oder der Retina selbst bedingten concentrischen Einschränkungen aus. 
peripheren Grenzen des Gesichtsfeldes durchaus kein conformes, sondern dieselben zeigten ein ganz erhebliches Hin- und Herschwanken, so dass es zuweilen zu einer Art Gegenbewegung kam, wenn ich mich dieses Ausdruckes bedienen darf, d. h. zu derselben Zeit, während welcher das Gesichtsfeld sich rom Centrum nach der Peripherie aufhellte, engte es sich in amgekehrtem Sinne ein. Ebenso können wir wohl für diesen Fiall behaupten, dass die concentrische Gesichtsfeldeinschränkung als Folge- und Begleiterscheinung des die Hemianopsie bedingenden Processes anzusehen ist.

Der Augenbefund ist in früheren Jahren ein durchaus normaler gewesen; auch jetzt zeigen weder Gesichtsfeld, noch Sehschärfe irgend eine Abweichung von der Norm. Es liegt also kein Grund vor zu der Annahme, dass etwa die concentrische Einsohränkung vor der Anfallsserie bestanden hat, und die Hemianopsie nur eine zufällige Complication gewesen ist.

Die Abhängigkeit der concentrischen Einschränkung von der die Hemianopsie bedingenden Störung ging ja zur Genüge aus dem schon mehrfach erwähnten Schwanken der peripheren Grenzen während des Bestehens der Hemianopsie hervor.

Erst nach dem Verschwinden der Hemianopsie sehen wir auch das Gesichtsfeld im Uebrigen sich allmälig zur Norm erweitern.

Endlich lässt sich für unseren Fall die interessante Thatsache constatiren, dass die Hemianopsie früher zum Ausgleich kam, als die sie begleitende concentrische Gesichtsfeldeinschränkung.

In wieweit hierin ein gesetzmässiges Verhalten zu erkennen ist, muss weiteren Beobachtungen vorbehalten bleiben.

Das Interesse des vorliegenden Falles, beruht also:

1. In der erstmaligen Beobachtung einer transitorischen Hemianopsie bei cerebraler Kinderlähmung;

2. In der Complication dieser Hemianopsie mit concentrischer Gesichtsfeldeinschränkung und

3. In dem gegenseitigen Verhalten dieser beiden in rerschiedenem Sinne functionellen Störungen während der Reevolution des Gesichtsfeldes. 